\title{
Magneto-Optical Studies of Quantum States of Two-Dimensional Hole Gas at Single $\mathrm{Ga}_{1-x} \mathrm{Al}_{x} \mathrm{As} / \mathrm{GaAs}$ Heterojunction
}

\author{
L. BRYJA \\ Institute of Physics, Wrocław University of Technology \\ Wybrzeże Wyspiańskiego 27, 50-370 Wrocław, Poland
}

\begin{abstract}
The detailed studies of low temperature $(T=2 \mathrm{~K})$ polarization resolved photoluminescence and photoluminescence excitation studies from heavily modulation doped $\mathrm{Ga}_{1-x} \mathrm{Al}_{x} \mathrm{As} / \mathrm{GaAs}$ single heterojunctions in continuous wave and time-resolved conditions are reported. The free conduction band GaAs electrons recombination with two-dimensional holes observed in low magnetic fields enabled us to determine experimentally the valence subband Landau levels. The high magnetic field photoluminescence spectra are dominated by excitonic transitions which are attributed to neutral excitons rather than to positively charged ones.
\end{abstract}

PACS numbers: 78.55.Cr, 71.70.Di

\section{Introduction}

Two-dimensional semiconductor structures have attracted, in recent years, much interest of both technologists and scientists. The structures are used in elaboration of high performance devices and also in the study of fundamental physics phenomena, e.g. integer and fractional quantum Hall effects $[1,2]$. Although optical properties of both $n$ - and $p$-type, two-dimensional (2D) structures have been intensively studied, the evolution of the optical response of these structures is not clearly understood. The Fermi edge singularity [3] and the formation of charged excitons [4] are two important effects to be mentioned in this context. A negatively charged exciton (also called trion) is an analogue of $\mathrm{H}^{-}$in semiconductors. It was predicted by Lampert [5] to be stable in bulk semiconductors but was not observed due to a very small binding energy. In quantum wells, with $2 \mathrm{D}$ electrons or holes, the binding energy of an additional carrier to a neutral exciton increases up to a few meV making negatively $\left(X^{-}\right)$and positively $\left(X^{+}\right)$charged excitons 
possible to detect in absorption and emission measurements $[3,6,7]$. The problem of trions at a single interface is controversial. The observation of negatively charged excitons have been reported in the study of photoluminescence (PL) from $\mathrm{Ga}_{1-x} \mathrm{Al}_{x} \mathrm{As} / \mathrm{GaAs}$ single heterojunctions [8], but the important problem not discussed in this article is the distinction between the character of negatively charged excitons which may be either free or localised, resembling in the latter case the conventional bound excitons. Also, the band to band transitions observed in photoluminescence from modulation doped $\mathrm{Ga}_{1-x} \mathrm{Al}_{x} \mathrm{As} / \mathrm{GaAs}$ single heterojunctions are qualitatively different than those observed in PL from quantum wells. While both photo-created carriers are localised in a quantum well they are separated in growth direction by electric field at a single junction. In heavily doped heterostructures with high densities of 2D carriers the strong electric field appreciably separates spatially recombining electrons and holes. As a result, the band to band transition has a free character in a low and medial magnetic field limit [9-11]. In this paper we report on the detailed studies of two-dimensional hole gas states at a single $p$-type doped $\mathrm{Ga}_{1-x} \mathrm{Al}_{x} \mathrm{As} / \mathrm{GaAs}$ interface using polarization resolved low temperature $(T=2 \mathrm{~K})$ photoluminescence and photoluminescence excitation (PLE) in continuous wave (cw) and time-resolved conditions. It will be shown that using low excitation power densities a very rich photoluminescence spectrum is revealed. From the analysis of well-known $H$-band emission [11], which is interpreted as a free carriers recombination with two-dimensional holes the energy dispersion of the valence band Landau levels (LL) is determined. The experimental results are compared with detailed numerical calculations based on our theoretical model [12]. In addition to free carrier lines, in low magnetic fields, recombination of donor bound electrons with $2 \mathrm{D}$ holes is observed. In higher magnetic fields new lines attributed to interface excitons are discerned in PL spectra.

\section{Samples parameters and experimental techniques}

We have studied photoluminescence from five modulation doped $p$-type $\mathrm{Ga}_{1-x} \mathrm{Al}_{x} \mathrm{As} / \mathrm{GaAs}$ single heterojunctions with different $2 \mathrm{D}$ holes concentrations: $p_{1}=9.8 \times 10^{11} \mathrm{~cm}^{-2}, p_{2}=7.6 \times 10^{11} \mathrm{~cm}^{-2}, p_{3}=4.2 \times 10^{11} \mathrm{~cm}^{-2}$, $p_{4}=3.1 \times 10^{11} \mathrm{~cm}^{-2}$, and $p_{5}=2.0 \times 10^{11} \mathrm{~cm}^{-2}$. They were grown by molecular beam epitaxy on (001) semi-insulating GaAs substrate using the following sequence of layers (samples 1 and 2): $1 \mu \mathrm{m}$ GaAs, $7 \mathrm{~nm}$ undoped $\mathrm{Al}_{0.5} \mathrm{Ga}_{0.5}$ As spacer, $50 \mathrm{~nm}$ Be-doped $\left(1 \times 10^{18} \mathrm{~cm}^{-3}\right) \mathrm{Al}_{0.5} \mathrm{Ga}_{0.5} \mathrm{As}$ and $5 \mathrm{~nm}$ Be-doped $\left(2 \times 10^{18} \mathrm{~cm}^{-3}\right)$ GaAs cap. Samples 3,4 , and 5 had $x=0.3$ aluminium mole fraction content, the much wider spacer varied from $20 \mathrm{~nm}$ to $30 \mathrm{~nm}$ and were carbon doped. The low temperature $(T=4.2 \mathrm{~K})$ mobility of samples 3,4 , and 5 was high, changing from $\mu=1.33 \times 10^{5} \mathrm{~cm}^{2} /(\mathrm{V} \mathrm{s})$ to $7.99 \times 10^{4} \mathrm{~cm}^{2} /(\mathrm{V} \mathrm{s})$. Mobility of samples 1 and 2 were smaller of about one order of magnitude. All measurements were performed in liquid helium temperature down to $2 \mathrm{~K}$ with a magnetic field applied 
perpendicular to the growth direction in Faraday configuration. Continuous wave photoluminescence and photoluminescence excitation measurements were carried out using Bitter solenoids providing magnetic fields up to 23 T. An optical fibber system was used with the quarter-wave plate and the linear polarizer placed together with the sample in the liquid helium. In order to examine the circular $\left(\sigma^{+}\right.$and $\left.\sigma^{-}\right)$polarization of the photoluminescence the direction of magnetic field was changed. The spectra were analysed in the $1 \mathrm{~m}$ single grating monochromator with nitrogen-cooled charged-coupled device (CCD) used as a detector. Time-resolved photoluminescence (TRPL) experiments were performed in optical split coil superconducting cryostat giving magnetic fields up to $7 \mathrm{~T}$. The spectra were analysed in the same way as in the first set up.

\section{Experimental results}

As we have shown previously in the study of photoluminescence from modulation doped single junctions a critical role plays the power density of applied laser excitation [10, 12]. Photo created electron-hole pairs are separated by interface electric field giving additional electric field of opposite direction which at strong excitation level considerably reduces the bend bending existing in dark conditions. In Fig. 1 the evolution of photoluminescence spectra as a function of excitation power density, for the samples 1 and 2 with the highest $2 \mathrm{D}$ hole concentrations are shown. When the excitation power density is higher than $P=10 \mathrm{~W} / \mathrm{cm}^{2}$ only

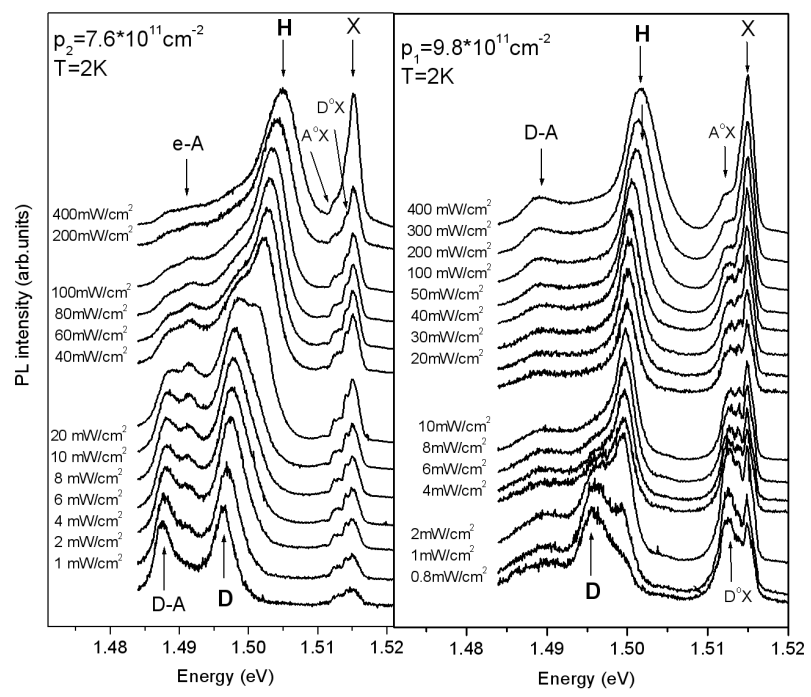

Fig. 1. Photoluminescence spectra for samples $p_{1}$ and $p_{2}$ in different excitation power densities. 
bulk GaAs excitons lines are discerned in the PL spectra (not presented). For excitations below this value a well-known $H$-band emerges on the low energy wing of bulk lines named $X, D^{0} X$, and $A^{0} X$ and attributed to free, donor-bound, and acceptor-bound GaAs excitons, respectively. As the excitation power is further reduced $H$-line intensity compared to the bulk ones increases. Simultaneously the $H$-band position shifts considerably to lower energies whereas positions of bulk transitions remain unchanged. For the excitation power lower than $10 \mathrm{~mW} / \mathrm{cm}^{2}$ for the sample 1 , and $100 \mathrm{~mW} / \mathrm{cm}^{2}$ for the sample 2 on the low energy side of $H$-band a new line which we named $D$-line emerges in the PL spectra. With a further reduction in laser power this line grows in intensity in comparison to $H$-band, which gradually disappears from the PL spectra and becomes invisible for excitations lower than $0.8 \mathrm{~mW} / \mathrm{cm}^{2}$ and $10 \mathrm{~mW} / \mathrm{cm}^{2}$ for samples 1 and 2 , respectively. The $D$-line exhibits the same red shift as the $H$-band with the decrease in laser power density. All measured PL intensities were normalised to obtain the same values at $H$-band or $D$-line maxima.

In order to obtain more information about the nature of observed lines we performed low temperature $(T=2 \mathrm{~K})$ polarization resolved photoluminescence in magnetic field up to $23 \mathrm{~T}$ applied perpendicular to 2D plane in Faraday configuration. The spectra have been collected with magnetic steps smaller or equal $0.2 \mathrm{~T}$, and their field evolution has been analysed with a high precision. We have ascertained that a further decrease in laser power is indispensable to attain a high resolution of PL measurements. This restricts, however, the observation of $\mathrm{H}$ band emission to higher magnetic fields but reveals the fine structure of emission related to 2D holes. We have noticed that $H$-band stops to shift towards lower energies and remain at the same position when the laser power density is decreased below $0.5 \mathrm{~mW} / \mathrm{cm}^{2}$ and $0.7 \mathrm{~mW} / \mathrm{cm}^{2}$ for samples 1 and 2 , respectively. This is evidence that at these power excitation levels photo created electron and hole pairs do not alter the interface potential. In Fig. 2 the photoluminescence spectra detected with application of very low excitation power $P=0.3 \mathrm{~mW} / \mathrm{cm}^{2}$ at filling factors $\nu=6$ and $\nu=3$ for the samples 1 and 2 are presented. The magnetic field dependence of the energy position of the observed interface-related lines and bulk $A^{0} X$ excitons are plotted in Fig. 3. In low magnetic field the photoluminescence spectra are dominated by $D$-lines recombination. The $H$-band emerges in the PL spectra for magnetic field higher than $1 \mathrm{~T}$ and $2 \mathrm{~T}$ for samples 1 and 2 , respectively. The intensity of $H$-band strongly increases while the $D$-line gradually disappear (not presented, see Refs. [10] and [12]). Both $H$-band and $D$-line split into four components in magnetic fields (two components observed in each circular polarization $\sigma^{+}$and $\sigma^{-}$). The line $D 4$ is not placed in Fig. 3, since it can be detected with an accurate precision only for a few magnetic fields. As it is clearly seen in Fig. 3, the $H$-band components change linearly with magnetic field while $D$-lines exhibit a diamagnetic shift which is evidence that a strong Coulomb interaction is involved in these transitions. After detailed analysis of experimental 


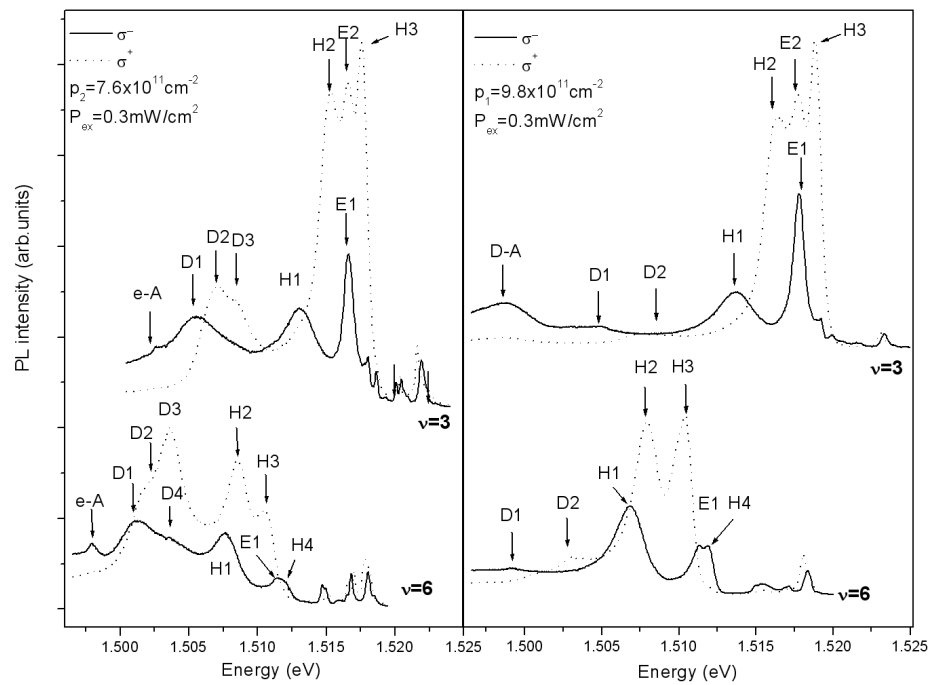

Fig. 2. Photoluminescence spectra from samples $p_{1}$ and $p_{2}$ in different circular polarization at the filling factors $\nu=6$ and $\nu=3$.

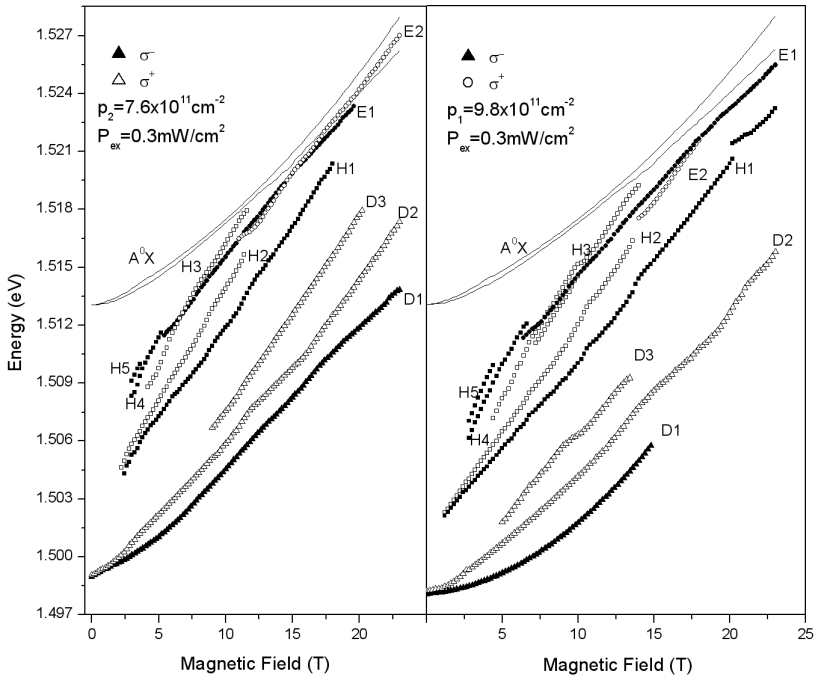

Fig. 3. Magnetic field evolution of interface related and bulk $A^{0} X$ photoluminescence for samples $p_{1}$ and $p_{2}$.

data and comparison with numerical calculations of interface potential and hole wave functions we have interpreted $D$-lines as recombination of electrons trapped on ionised donors near the interface with $2 \mathrm{D}$ holes confined at interface [10]. The $H$-band lines were attributed to recombination of free GaAs conduction band electrons with the same 2D holes. Therefore, the magnetic field evolution of the 

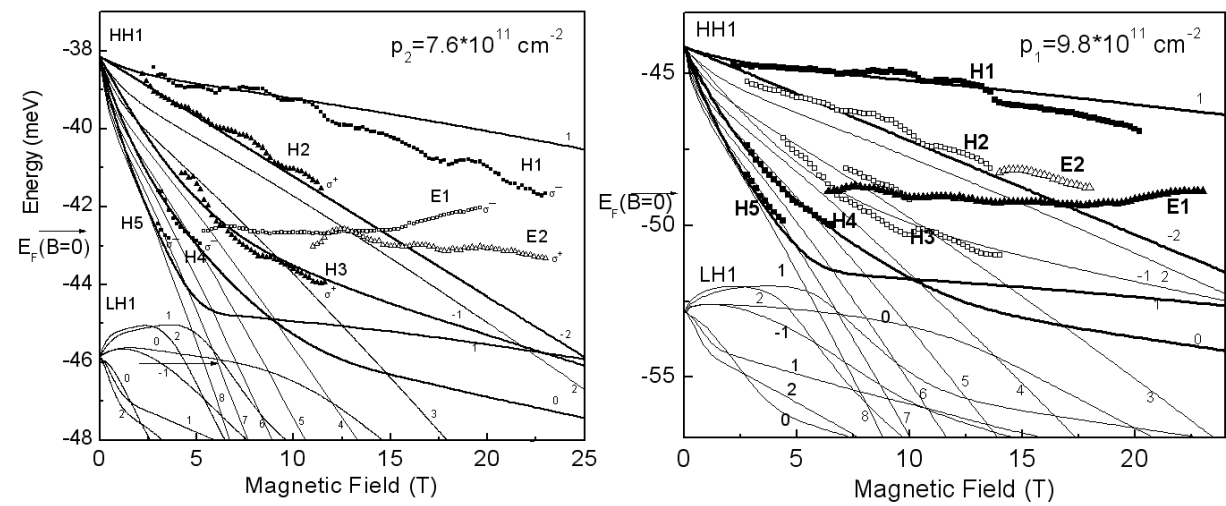

Fig. 4. The $2 \mathrm{D}$ holes Landau level dispersion in the magnetic field for samples $p_{1}$ and $p_{2}$ (symbols - experiment, lines - theory).

$H$-band allowed us to determine 2D hole Landau levels in the valence subband. This can be obtained by subtracting the measured transition energies from the energy $E_{\mathrm{cb}}+0.5 \hbar \omega_{\mathrm{c}} \pm 0.5 \mu_{\mathrm{B}} g B$ of a GaAs conduction band electron in the lowest Landau level. Here, $\mu_{\mathrm{B}}$ is the Bohr magneton, $m_{\mathrm{e}}=0.067 m_{0}$ is an electron effective mass, $g=-0.44$ is Lande factor of an electron and $\omega_{\mathrm{c}}=e B / m_{\mathrm{e}}$ is an electron cyclotron frequency. The energy $E_{\mathrm{cb}}$ was used as a fitting parameter. For each transition the sign of the Zeeman energy in the total energy of an electron was determined on the base of theoretical calculations [12]. The comparison of experimental data with detailed numerical calculations, for the samples 1 and 2, are presented in Fig. 4. A very good agreement is obtained for magnetic fields up to a filling factor $\nu=3$. The disagreement between energy positions of experimentally obtained and calculated numerically $H 1$ positions in higher magnetic fields is not resolved in this state of study. The possible explanation of this discrepancy can be attributed to the hole-hole interaction and the change of interface potential shape caused by redistribution of $2 \mathrm{D}$ holes. As it is seen in Figs. 2, 3, and 4 in the energy range of $H$-band transitions we observed lines, named by us $E 1$ and $E 2$, which do not coincide with any Landau levels. The line $E 1$ appears in PL spectra in $\sigma^{-}$polarization at the low energy side of the line $H 4$, which in turn dramatically disappears from the PL spectra when the 2D hole Landau level attributed to this transition crosses the Fermi level at magnetic field corresponding to a filling factor $\nu=6$. As the magnetic field is increased this line strongly grows in intensity (see Fig. 5). The similar behaviour is observed in PL spectra for lines $E 2$ and $H 3$ in $\sigma^{+}$polarization in the magnetic field higher than those corresponding to a filling factor $\nu=3$. According to our calculations, lines $H 3$ and $H 4$ are related to the recombination of photo excited electrons with 2D light holes [12]. Therefore, we attributed lines $E 1$ and $E 2$ to light hole excitons recombination. For the samples 3,4 , and 5 we have not discerned such rich structure of photoluminescence spectra and distinction between $H$-band and $E$-lines is not possible. We observed only 
continuous change of the magnetic field energy dependence of interface related recombination, from linear $(H$-band) to diamagnetic $(E$-lines) one with an increase of the field. This transition is observed similarly as for samples 1 and 2 near the filling factors $\nu=6$ and $\nu=3$ for polarization $\sigma^{-}$and $\sigma^{+}$, respectively. There is a question about the exact nature of $E$-lines. In the case of narrow quantum wells with a high 2D particle density, only charged excitons are observed. Theoretical calculations exclude, however, the creation of charged excitons in strongly asymmetric structures [13]. Detailed studies of lines $E 1$ and $E 2$ in PL spectra in higher magnetic fields under continuous wave excitation are complicated in our heterostructures by a dramatic increase in intensities of recombination attributed to bulk GaAs neutral acceptor bound excitons $\left(A^{0} X\right)$, which is clearly seen in Fig. 5. In all studied samples $A^{0} X$ lines exceed in intensities lines $E$ in higher

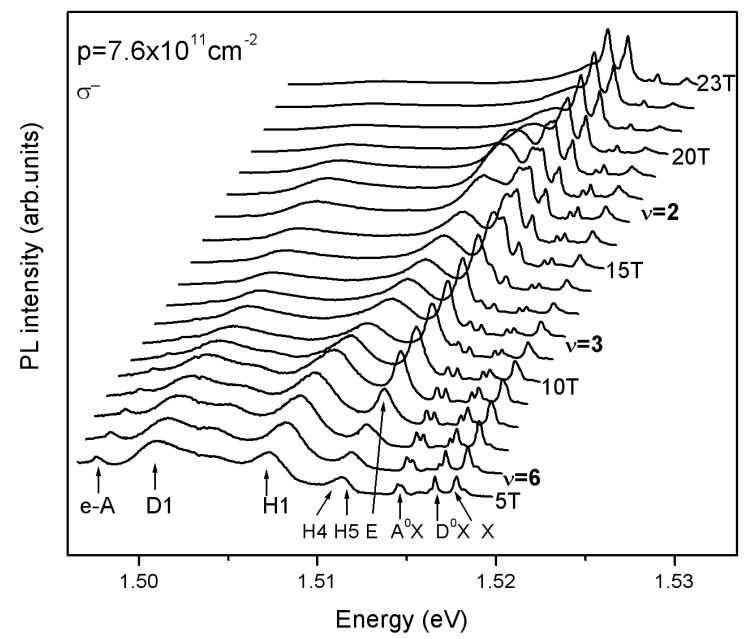

Fig. 5. Photoluminescence spectra evolution for the sample $p_{2}$ in $\sigma^{-}$circular polarization.

magnetic fields. In PL spectra of samples 3, 4, and 5 there is even an inversion in the ratio of their intensities. To omit this difficulty we performed time-resolved photoluminescence measurements in magnetic field up to $7 \mathrm{~T}$ in the same experimental conditions as continuous wave ones. The TRPL studies were performed with the use of $3 \mathrm{KHz}$ Nd-YaG laser with $10 \mathrm{~ns}$ impulse and CCD gated camera used as a detector. The time evolution of photoluminescence spectra for samples with high $\left(p_{2}=7.6 \times 10^{11} \mathrm{~cm}^{-2}\right)$ and the lowest $\left(p_{5}=2 \times 10^{11} \mathrm{~cm}^{-2}\right)$ concentrations are presented in Fig. 6. The continuous waves PL spectra are also placed for comparison. As we suppose the GaAs exciton related transitions disappear from the PL spectra recorded in ns delay time which allows the observation of interface related transitions. For all studied samples we detect a very long time of photoluminescence of $E$ and $H$ lines which is evidence of a low value of oscillator 


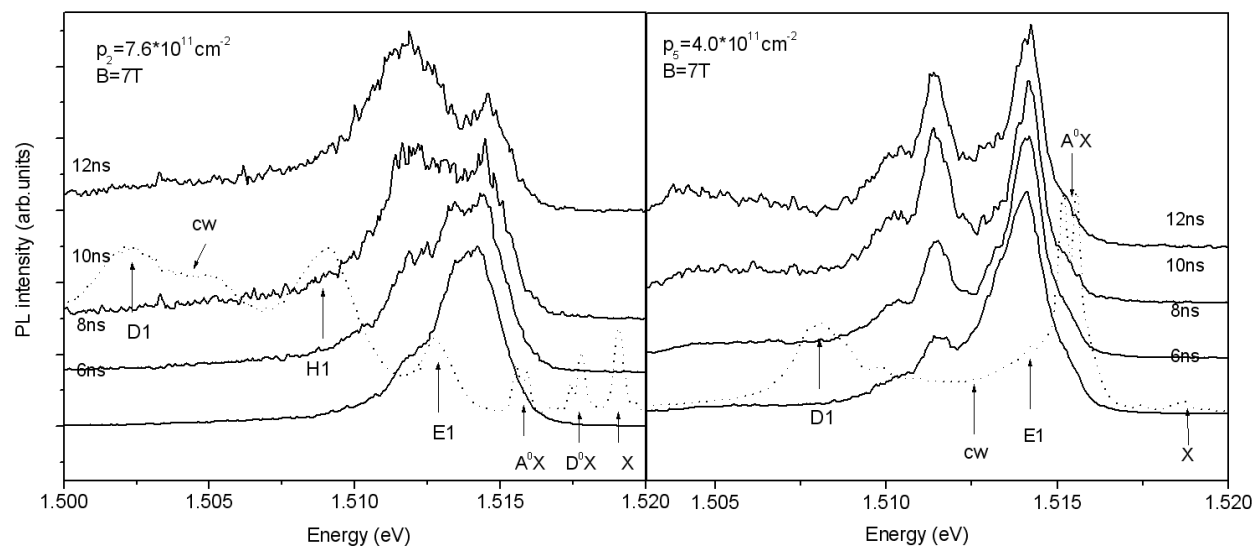

Fig. 6. The evolution of time-resolved photoluminescence spectra for samples $p_{2}$ and $p_{5}$ in the magnetic field $B=7 \mathrm{~T}$.

strengths of these recombinations. To confirm this observation we performed photoluminescence excitation measurements. In the PLE experiments we recorded the whole PL spectra using CCD camera. As a light source we used a tuneable Ti-sapphire laser. The spectra were collected with a laser wavelength step smaller or equal $0.25 \mathrm{~nm}$ varied in energy from the donor acceptor recombination up to the energy greater than the GaAs energy gap. An extremely low laser power and exposure time were used in order to prevent the CCD device from damage. The photoluminescence spectra for samples 2 and 5 are presented in Fig. 7. In the lower part of the figure the PL spectra for laser energy higher than GaAs

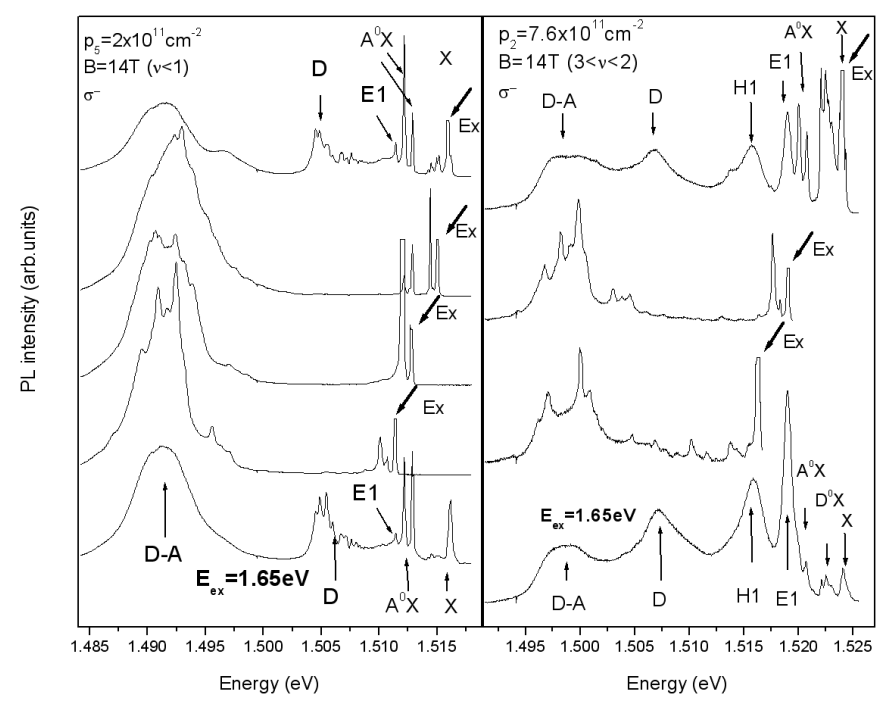

Fig. 7. Photoluminescence spectra for samples $p_{2}$ and $p_{5}$ for several excitation energies. 
energy gap is placed. In the upper part a few photoluminescence spectra with excitation energies at characteristic PL lines (marked by arrows) are shown. As it is clearly seen the interface related lines $(D, H$, and $E$ ) appear is higher than $P=10 \mathrm{~W} / \mathrm{cm}^{2}$ only bulk GaAs excitons lines are discerned in the PL spectra (not presented). For excitations in the PL spectra only when laser energy is equal or higher than the energy of a free GaAs bulk exciton. When the laser excitation is lower only the fine structure of the resonant excitation of donor acceptor recombination $(D-A)$ is observed. Thus, the photoluminescence excitation experiments also exhibit the considerably high space displacement of photo excited electrons and $2 \mathrm{D}$ holes. In numerical calculations we obtained that this displacement in zero a magnetic field, for the sample 2, is higher than $40 \mathrm{~nm}$ [10]. The magnetic field applied perpendicular to 2D plane squeezes the electron wave function and brings electrons closer to confined holes but a spatial separation between electrons and holes still remains larger than the distance between holes. Thus, the repulsion between 2D holes exceeds the attraction between an electron and an extra hole and positively charged excitons are not created.

\section{Conclusions}

We investigated photoluminescence from $p$-type doped single interfaces. We showed that detailed polarization resolved PL measurements under the application of very low excitation power densities explain discrepancies in the interpretation of interface related recombination of the so-called $H$-band. In the study of heavily doped heterostructures with 2D holes concentrations higher than $p=7.6 \times 10^{11} \mathrm{~cm}^{-2}$ in low and medial magnetic fields the free-carrier recombination with $2 \mathrm{D}$ holes is discerned in the photoluminescence spectra which is consistent with previous observations [9-11]. These transitions enabled us to determine experimentally the energy of $2 \mathrm{D}$ holes Landau levels. In higher magnetic fields exciton lines appear in the PL spectra. The distinction between free and bound recombination in PL spectra is possible only for the heterostructures with the highest $2 \mathrm{D}$ hole concentration. However, even for samples with lower 2D hole concentrations down to $2 \times 10^{11} \mathrm{~cm}^{-2}$ the free-carrier recombination is observed in PL spectra in low magnetic fields. We interpreted exciton transitions as recombination of electrons with 2D light-holes which occupy states below the Fermi level. This is consistent with the interpretation of $H$-band proposed by Shen et al. [14] as interface excitons without any contribution of 2D holes.

\section{Acknowledgments}

This work was performed in collaboration with M. Kubisa, K. Ryczko, J. Misiewicz, G. Ortner, M. Kneip, M. Bayer, D. Reuter, A. Wieck, M. Byszewski, R. Stȩpniewski, and M. Potemski. 


\section{References}

[1] K. von Klitzing, G. Dorda, M. Pepper, Phys. Rev. Lett. 45, 494 (1980).

[2] H.L. Stormer, D.C. Tsui, A.C. Gossard, Rev. Modern Phys. 71, 298 (1999).

[3] M.S. Skolnick, J.M. Rorison, K.J. Nash, D.J. Mowbray, P.R. Tapster, S.J. Bass, A.D. Pitt, Phys Rev. Lett. 58, 2130 (1987).

[4] K. Keng, R.T. Cox, Y. Merle d'Aubigné, F. Bassani, K. Saminadayar, S. Tatarenko, Phys. Rev. Lett. 71, 1752 (1993).

[5] A. Lampert, Phys. Rev. Lett. 1, 450 (1958).

[6] G. Finkelstein, H. Shtrikman, I. Bar-Ioseph, Phys. Rev. Lett. 74, 976 (1995).

[7] J. Shields, M. Pepper, D.A. Ritchie, M.Y. Simmans, G.A.C. Jones, Phys. Rev. B 51, 18049 (1995).

[8] Y.F. Kim, F.M. Munteanu, C.H. Perry, D.G. Rickel, J.A. Simmons, L.N. Pfeiffer, K.W. West, Phys. Rev. B 64, 195302 (2001).

[9] W. Ossau, E. Bengert, G. Weimann, Solid State Commun. 64, 711 (1987).

[10] L. Bryja, M. Kubisa, K. Ryczko, J. Misiewicz, A. Larionov, M. Bayer, A. Forchel, C.B. Sorensen, Solid State Commun. 122, 379 (2002).

[11] Y.R. Yuan, K. Mohammed, M.A.A. Pudensi, J.L. Merz, Appl. Phys. Lett. 45, 739 (1984).

[12] M. Kubisa, L. Bryja, K. Ryczko, J. Misiewicz, C. Bardott, M. Potemski, G. Ortner, M. Bayer, A. Forchel, C.B. Sorensen, Phys Rev. B 67, 35305 (2003).

[13] I. Szlufarska, A. Wójs, J.J. Quinn, Phys. Rev. B 63, 85305 (2001).

[14] J.X. Shen, Y. Oka, C.Y. Hu, W. Ossau, G. Landwehr, K.J. Friendland, R. Hey, K. Ploog, Phys. Rev. B 59, 8093 (1999). 